\title{
How Malicious Comments on Social Media Has a Huge Impact on the Negative Attitude Toward Women
}

\author{
Puiyu Huang \\ Basis International School Shenzhen, Shenzhen, Guangdong 518067, China, puiyuhuang0719@163.com
}

\begin{abstract}
In this age of the Internet, using social software has become an indispensable part of our lives. Although social software allows us to share our daily life, there will always be negative comments on social media. Since these comments are very common on social media, some people may think that these bad comments will only make the publisher's mood down for a moment. In fact, this small factor may cause some bigger changes to the user's mind like the butterfly effect. The main focus of this research paper is how these negative review on social media affects sexual double standards. The prediction of this research is that these bad reviews will cause sexual double standard to a certain extent. So, when they do the IAT test after reading the bad reviews, it will cause the D score to drop. Of course, there are many imperfections in this research, so future research can focus on the correlation between SDS and social media more extensively. The implication of this research is to find out possible causes and solution of SDS, and further build a positive environment on social media.
\end{abstract}

Keywords: sexual double standards, social media, cognitive learning theory, negative stimuli, Implicit association test.

\section{INTRODUCTION}

Psychologists have developed various theories in order to explain the causes of SDS. Sexual double standard, by definition, means that men and women are judged differently for the exact same sexual behaviors. Many evolutionary psychologists suggest these biases are based on inborn differences between men and women. For example, Female can make sure their offspring carry their gene, because they give birth to them. However, men cannot be certain whether he is really the parent of offsprings [1]. This explains why people show more tolerance to a man who have many sexual partners compared to women. Although women are granted more freedom nowadays, these variations still exist in current society. This caused the development of theories like social role and social script theories [2,3]. These two theories illustrate that SDS is caused by different social expectation of men and women. Since the society have different expectation for men and women, people would have contrasted attitude toward men and women when discussing sexual activities. There is long been a controversy over whether SDS really exists. According to a survey conducted by Marks (2002), about $85 \%$ of people suspect sexual double standard is observable in our society [4]. However, there aren't consistent results of SDS's for proving its existence. Both men and women will be viewed negatively if they engage in casual sexual activities, but differences between people's attitude are not seen. [5]. Also, there are more and more factors like social interaction and social media that need to be add into our considerations. These are limitation of earlier studies. An early study focuses on social interaction and peer influences. This research concluded that family and peer interaction would cause D score to increase in an explicit SDS test especially during adolescence. [6]. Moreover, it is worth to mention that media have increasing impact on SDS. According to a TV guide [7], media would reinforce stereotypical and negative views about women's sexual behaviors. This phenomenon is becoming more obvious when it gets exposed in social media. Since a man would be viewed as successful if he had sex with many women but women are likely to be seen as slut if they engage in same level of sexual activity as men [8], then the effect of these negative and positive comments on SDS will be amplified when putted in social media. The research question is how malicious comments on social media that serve as punishment has a huge impact on the negative attitude toward women. 


\section{PRESENT WORK}

The research question is inspired by all theories I discussed above, but my logic is mainly based on cognitive social learning theory. Cognitive social learning theory is the variation in rewards and punishments men and women received after illustrating their engagement in sexual behaviors is one of the main causes of SDS [9]. The prediction of the study is that SDS would increase after negative comments are added to the post. Since the fact that women's sexual partners cannot exceed men's sexual partners became an unspoken norm, women will think their sexual life is not allowed or reasonable after these negative comments. This would shape women's thoughts about unfair differences between men and women regarding to sexual life. Overall, if SDS does increase (more negative) as predicted, then we can conclude that negative comments did serve as a positive reinforcement to existing sexual biases. It is worth to investigate because social media is dominating our daily life, and negative comments are a common thing in social media. If negative comments have huge impacts on SDS, then these comments should be prohibited to a certain extent.

\section{METHOD}

\subsection{Materials}

First of all, a social media platform is essential to our study. Researchers would need to cooperate with programmers. There are few requirements for the social media platform. Jan H.Kietzmann suggests that there are seven fundamental blocks in social media [10]. For our research purpose, three of the seven blocks should be available. Participants should be able to share their sexual life, and they should know the existence of other users (researchers). Also, they should reveal their own behaviors, and show their own identity by posting their sexual life. The other crucial measurement in the research is the IAT test. The implicit Association Test is used to test attitudes, prejudices, and stereotypes. It is essential to implicit measurement of Sexual Double Standard because it reveal the higher validity measures of SDS, instead of low validity of explicit measures [11].The IAT test consist of a set of obvious positive words, and a set of obvious negative words. There are also target sets, which are what we are interested during the test. There will be two letters corresponding to positive and negative. Participants will need to press a letter accordingly to given stimuli. If the target word is more associative with positive in participants mind, then they will react faster to the target word and will be able to click the represented letter faster. If not, they will react slower [12]. IAT test for our research will be categorized into two steps. First, participants will need to categorize whether the image shown on the screen is sexually positive or sexually negative images. Then, our target words should be names of male and female. According to the prediction of our research, participants (after negative comments are given) would react longer when a female name is associated with sexual positive images. Similarly, they will react faster when the female name is associated with sexual negative images. Positive sexual images will be full of passion and enjoyment. Whereas negative sexual images will be abusive and violence. All names will be either very feminine or masculine. There will be four blocks. Participants will be able to practice step one in the first block. They will understand how to categorize images to sexual positive or sexual negative categories. Also, participants will be able to practice step two in the second block. They will be able to recognize whether the name is a woman's name or a man's name. This step is inevitable because this step will make sure participants will show their attitude toward woman clearly and directly. These two steps are only for practical purposes, so data will not be collected. Therefore, data collection will happen in step three and four. Step three and four is technically the same thing. However, their direction of choices will change. For block three, If the It is a positive set, then the participants should click on "a". If it belongs to the negative set, then the participants should click on "l". For block four, their order will change. This means that letter "a" stands for negative set, and "l" will be corresponded to positive stimuli. This design will eliminate the difference between right-handed and left-handed [9].

\subsection{Participants}

Participants would be 500 adolescences (all females). They will enroll in the social media that are designed for this study. This design is reasonable because SDS itself is based in negative attitude towards women's sexual behavior. Plus, the research question is how these negative comments would affect women's SDS. In this case, no male participants are reasonable. This study focuses mainly on the change within a person in SDS, so individual or cultural differences can be ignored. The reason why the participants are all adolescence because this is a critical period where the participants will develop their own cognitive and social skills [13]. So, their behaviors will span across her lifetime and would influence the next generation. This means that their data will represent larger amount of people. [12].

\subsection{Procedure}

Participants would be recruited and do the following procedure. All participants will get in to our social media. That social media is designed only to test the research question. This social media should not be open to anyone who are not involved in this study. Participants are responsible to post their own and accurate sexual life in the social media. 


\section{Measure 1}

For step 1, researchers would comment neutrally to their life. Neutrally comments are those comments that does not support or disprove participants' post. In these circumstances, their D score should be considered as their basic D score. After that (step two), these same participants would enter step 2 . The time between step 1 and step two should not exceed five minutes. If this time gap is not controlled, then there might be another factor influencing SDS. Or the effect of these stimuli (comments) will not function efficiently.

\section{Measure 2}

For step 2, they will be asked to post something related to their sexual activity (similar to posts they posted in step one). However, researchers would respond to their post negatively. After that, these same group of participants would be asked to do IAT test again. Their final SDS result will be obtained and used to analyze the result. Researchers would not need to deceive participants when taking IAT test, because the research only focuses on implicit sexual double standard. In this case, even if they know they are going to do a SDS test later, the results of this research will not vary. Also, by measuring implicit sexual double standard, social desirability theory would not occur in the study. This means that participants' SDS result will be more objective. However, participants should not be able to know who posted these negative comments. If they know these comments were posted by researchers, they may not feel that much of negativity. This would affect our final outcome of D score, and our process of shaping their attitudes (cognitive social learning theory).

\section{RESULTS}

The mean of basic SDS will be calculated, and the mean of the new SDS will also calculated. These two results will be compared. In order to do that, we will use the t-test. The reason why we use T-test is because this research is a within study, and T-test is the most efficient measure when comparing two mean (step 1 and step 2). If SDS gets lower (D score increased), then it means their attitude is more positive. If SDS gets higher (D score decreased), then my hypothesis will be proven.

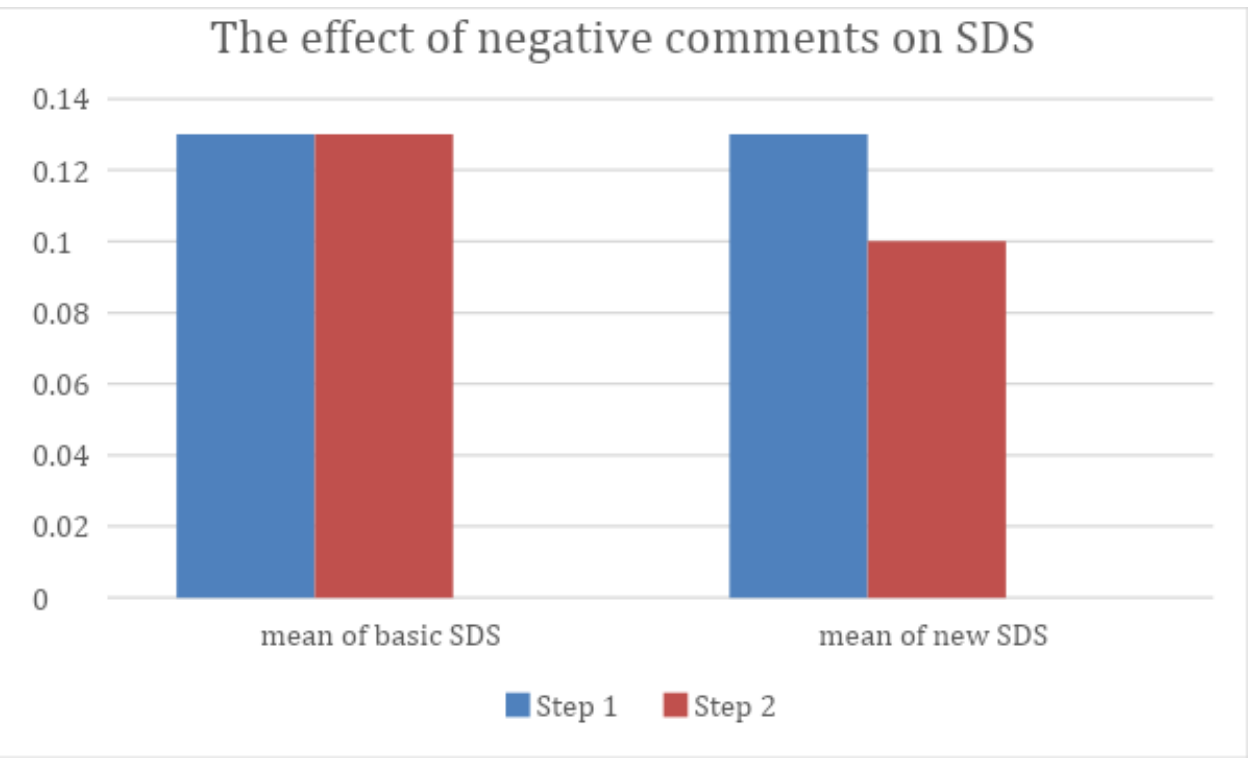

Figure 1 Descriptive statistics

According to Thompson, average female SDS is about 0.13 [9]. Although our research did not include specific measurements, figure 1 showed our predicted results. For the first step, which contains only neutral comments, will have no impact on D score. On the other hand, the D score for step 2, which contains only negative comments, will decrease D score. This indicates a more negative attitudes toward women who engage in sexual behaviors. The $\mathrm{P}$ value will probably be less then 0.05 , because the sample size is not small, and it based on the change of SDS. It is less likely that other stimuli will be the cause of the change in D score in this short time period. In this case, the result of the research is significant.

\section{CONCLUSION}

\subsection{Implication}

If the actual data proved the prediction of this study right, then we should encourage fewer negative comments on social media. If further study shows that positive comments will increases $\mathrm{D}$ score, then we now find a new way to decrease sexual double standard score. This study also points out how social media really shaped our value consciously and unconsciously. Hopefully, this research will also show how positivity in social media is essential. 


\subsection{Limitations and Future Directions}

Previous research use subconscious stimuli like priming to observe the impact of the stimuli [9]. However, this reveals one limitation of this study. The stimuli before IAT test are obvious. This research only focuses on how negative comments affect a specific gender and age group's D score. This is a limitation of my study because participants may notice the relationship between these negative comments with $\mathrm{D}$ score. If this happens, then Hawthorne effect would happen.

Future studies can focus on how positive comments on social media affect women's SDS. This research is limited to adolescence, so the result may vary if the participants are adult, or old people. Since adults already developed their own believe and interpretation about sexual behaviors, their change in SDS may not change a lot. So how to change adult's SDS will be a possible question for future studies. Furthermore, this research only focused on cognitive social learning theory, but there are far more theories about SDS. Since social media's influence are skyrocketing, future study can combine early theories with some other interesting phenomenon on social media. This research also only focuses on implicit SDS, future research can also focus on how to create a test to minimize biases when doing an explicit SDS test.

\section{REFERENCES}

[1]. Buss, D. M., Larsen, R. J., Westen, D., \& Semmelroth, J. (1992). Sex differences in jealousy: Evolution, physiology, and psychology. Psychological Science, 34), 251-256. doi:10.1111/j.1467-9280.1992.tb00038.x

[2]. Gaunt, R. (2012). Breadwinning moms, caregiving dads: double standard in social judgments of gender norm violators. J. Fam. Issues 20, 1-22. doi: $10.1177 / 0192513 \times 12438686$

[3]. Farvid, P., Braun, V., and Rowney, C. (2017). 'No girl wants to be called a slut!': women, heterosexual casual sex and the sexual double standard. J. Gender Stud. 26, 544-560. doi: 10.1080/09589236.2016.1150818

[4]. Marks, M. J. (2002). [Internet survey of attitudes of sexual free- dom.] Unpublished raw data.

[5]. Gentry, M. (1998). The sexual double standard. The influence of number of relationships and level of sexual activity on judg- ments of women and men. Psychology of Women Quarterly, 22, 505-511.

[6]. Cislaghi, B., Bhatia, A., Li, M., Lian, Q., Baird, S., Kayembe, P., ... \& Moreau, C. (2021). Changes in the Sexual Double Standard Associated With
Sociodevelopmental Factors Among Young Adolescents in Kinshasa. Journal of Adolescent Health, 69(1), S23-S30. https://doi.org/10.1016/j.jadohealth.2020.07.041

[7]. Waggett, G. J. (1989, May 27). A plea to the soaps: Let's stop turn-

ing rapists into heroes. TV Guide, pp. 10-11.

[8]. Barash, D. P., \& Lipton, J. E. (2001). The myth of monogamy. New York: Freeman. [9]. Thompson, A. E., Harvey, C. A., Haus, K. R., \& Karst, A. (2020). An investigation of the implicit endorsement of the sexual double standard among u.s. young adults. Frontiers in Psychology, 11. doi:10.3389/fpsyg.2020.01454

[10].Kietzmann, J. H., Hermkens, K., McCarthy, I. P., \& Silvestre, B. S. (2011). Social media? Get serious! understanding the functional building blocks of social media. Business Horizons, 54(3), 241-251. doi:10.1016/j.bushor.2011.01.005

[11]. Rezaei, A. (2011, May 23). Validity and reliability of the IAT: Measuring gender and ethnic stereotypes. Retrieved August 08, 2021, from https://www.sciencedirect.com/science/article/pii/S 074756321100094X? casa token=kWE7k5THhU8 AAAAA\%3AtSrJqETMwVtIT4W2X_CsbtFTjdqi XzwY99Bzr2KRUDrPSVQcP7nA7ogcYLd3 Wawpucx-mR-V2qr

[12].Brendl, C. M., Markman, A. B., \& Messner, C. (2001). How do indirect measures of evaluation Work? Evaluating the inference of prejudice in the Implicit ASSOCIATION TEST. Journal of Personality and Social Psychology, 81(5), 760-773. doi:10.1037/0022-3514.81.5.760

[13].Larsen, B., \& Luna, B. (2018, September 07). Adolescence as a neurobiological critical period for the development of higher-order cognition. Retrieved August 25, 2021, from https:/www.sciencedirect.com/science/article/abs/ pii/S014976341830160X 